\title{
ON HOMEOMORPHISMS OF INFINITE DIMENSIONAL BUNDLES. III
}

\author{
BY
}

\author{
T. A. CHAPMAN( 1 ) AND R. Y. T. WONG( (2)
}

ABSTRACT. In this paper we continue the study of homeomorphisms and prove an analogue of the homeomorphism extension theorem for bundles modeled on Hilbert cube manifolds; thus we generalize previous results for $Q$-manifolds (Anderson-Chapman). This analogy, as in the case of manifolds, requires a consideration of proper maps and proper homotopies. The approach to the present problem is similar to that considered in our previous papers. Bear in mind several distinct difficulties occur in our setting.

1. The purpose of this paper is to continue the study of homeomorphisms in the setting of bundles modeled on Hilbert cube manifolds (that is, a connected separable metric space having an open cover by sets homeomorphic to open subsets of the Hilbert cube $Q=[-1,1]^{\infty}$ ). Similar theorems are obtained under the following headings: characterization, separation of sets, mapping replacements and extending homeomorphisms. In the present setting we consider proper maps and proper homotopies. A map $f: X \rightarrow Y$ is proper provided the inverse images of compact sets in $Y$ are compact sets in $X$. A homotopy $F=\left\{f_{t}\right\}: X \times I \rightarrow Y$ is a proper bomotopy provided the map $F$ is proper.

Hypothesis. Throughout the following let $M$ denote a connected $Q$-manifold and, for matter of convenience, assume that all spaces considered are metrizable.

Our notation and definitions follow that of [7] and [8].

2. Separation of sets. We recall that for a bundle $E=(E, p, B)$ and for a pair $(U, \mathcal{U})$ denoting an open set of $E$ and an open cover of $U$, a $(U, \mathcal{U})$-isotopy $\left\{\mu_{t}\right\}$ on $E$ is a map $\mu=\left\{\mu_{t}\right\}: E \times I \rightarrow E$ such that $\mu_{0}=i d$, each $\mu_{t}$ is an isomorphism with support in $U$, and $\left\{\left.\mu_{t}\right|_{U}\right\}$ is limited by $\mathcal{U}$. A closed set $K$ in the product space $X \times Y$ is an $X$-projective $Z$-set provided the projection of $K$ into $X$ is contained in a closed $Z$-set of $X$. A closed subset $K$ of $E$ is a fibre $Z$ set provided $K \cap p^{-1}(b)$ is a $Z$-set in $p^{-1}(b)$ for each $b \in B$. The purpose of this section is to prove the following theorem.

Received by the editors May 4, 1971 and, in revised form, July 24, 1973. AMS (MOS) subject classifications (1970). Primary 57A20; Secondary 55F 10.

Key words and phrases. Hilbert cube manifolds, bundles, $B$-preserving homeomorphism, extension, mapping replacement, separation.

(1) Research supported in part by NSF 14429.

(2) Research supported in part by NSF Grant GP20632. 
Theorem 2.1. Let $E=(E, p, B)$ be given over a polybedron $B$ with fibre $M$. Suppose $K, L_{1}, L_{2}, \cdots$, are closed fibre Z-sets of $E$; then there is an isotopy $\left\{g_{t}\right\}$ of $E$ such that $g_{0}=$ id and $g_{1}(K) \cap\left(\bigcup_{i \geq 1} L_{i}\right)=\varnothing$.

Moreover, if $\mathcal{U}$ is any open cover of $K$, we may choose $\left\{g_{t}\right\}$ to be limited by U.

To give a proof we need the following lemmas.

Lemma 2.2. Let $E=(B \times M, p, B)$ be given over any space $B$ and let $K, L$ be closed M-projective Z-sets of $E=B \times M$. Suppose $K \backslash L \neq \varnothing$; then for any open cover $\mathcal{U}$ of $K$, there is a fibre homotopy $G=\left\{g_{t}\right\}: K \rightarrow E$ limited by $\mathcal{U}$ such that (1) $\left.g_{t}\right|_{K \cap L}=\mathrm{id}$ for all $t,(2) G(K \times I)$ is an M-projective Z-set, and (3) $g_{1}$ is a closed imbedding sucb that $g_{1}(K \backslash L) \cap(K \cup L)=\varnothing$.

Moreover, if $M=Q$ and $K \subset B \times s$, we may choose $G$ so that $G(K \times I) \subset B \times s$.

Proof. This follows from the Anderson-McCharen technique [5, $\$ 2]$ with minor modification.

Lemma 2.3. Let $E=(B \times Q, p, B)$ be given over compact polybedron $B$ and let $K_{0}, K_{1}$ be closed fibre Z-sets in $E$. Then there is an isotopy $\left\{b_{t}\right\}$ on $E$ such that $b_{0}=\mathrm{id},\left.b_{t}\right|_{K_{0}}=\mathrm{id}$ for all $t$ and $h_{1}\left(K_{1} \backslash K_{0}\right) \cap\left(K_{0} \cup K_{1}\right)=\varnothing$. Moreover, if $\mathcal{U}$ is any open cover of $K_{1}$, we may choose $\left\{b_{t}\right\}$ so that $\left\{\left.b_{t}\right|_{K_{1}}\right\}$ is limited by $\mathcal{U}$.

Proof. The proof follows more or less the same pattern as Lemma 3.9 of [7]. We outline as follows. We say $D$ is a standard k-cell in $s \subset Q$ if $D$ can be written in the form $D=\Pi_{i=1}^{k}\left[a_{i}, b_{i}\right] \times(0,0, \ldots)$ where $-1<a_{i}<b_{i}<1$ and $\left[a_{i}, b_{i}\right]$ is contained in the $i$ th-coordinate $J_{i}$ of $s$. It follows from Lemma 3.8 of [7] that, for any open set $U$ of $E$ containing $B \times D$ and for any $\epsilon>0$, there is an isomorphism $f$ of $E$ supported in $U$ such that $f(B \times s)=B \times s, d(f$, id $)<\epsilon$ and $f(K) \cap(B \times D)=\varnothing$, where $K=K_{0} \cup K_{1}$.

By an analogue of a procedure used in Lemma 7.1 of [2], the above statement implies that there is an isomorphism $g$ of $E$ such that $g(K)$ is a $Q$-projective $Z$-set. Let $\mathcal{O}$ be an open cover of $g\left(K_{1}\right)$ such that $\mathrm{St}^{(4)}(\mathcal{O})$ refines $g(\mathcal{U})$ (the open cover induced by $g$ and $\mathcal{U}$ ). With respect to the map $\left.g\right|_{K}: K \rightarrow E$ and to the open cover $\mathcal{O}$ of $g\left(K_{1}\right)$, by Lemma 2.2 there is a homotopy $G=\left\{g_{t}\right\}$ of $K_{1} \times I$ into $E$ such that $g_{1}\left(K_{1} \backslash K_{0}\right) \cap g(K)=\varnothing$. Using other properties of $G$ provided by Lemma 2.2 and Lemma 3.7 of [7], there is a $\left(U, \mathrm{St}^{(3)}(\mathcal{O})\right)$-isotopy $\left\{\mu_{t}\right\}$ on $E$ such that $\mu_{1} g_{0}=g_{1}$, where $U=\bigcup\{V \in \mathcal{O}\}$. $\left\{b_{t}=g^{-1} \mu_{t}\right\}$ is a desired isotopy.

Proof of Theorem 2.1. First suppose that $E$ is trivial and $B$ compact. Let 
us illustrate a procedure that will separate $K$ from $L_{1}$. Let $\left\{b_{i}\right\}$ be a sequence of imbeddings of $Q$ into $M$ such that the union $\bigcup_{i \geq 1} b_{i}(Q \backslash W)$ is an open cover of $M$, where $W=\left\{\left(x_{i}\right) \in Q: x_{1}=1\right\}$. We may assume that the sequence $\left\{U_{i}=\right.$ $\left.b_{i}(Q \backslash W)\right\}$ is ordered as in [4, Theorem 2]. Applying Lemma 2.3 there is a B-preserving isotopy $\left\{g_{1 t}\right\}$ of $B \times b_{1}(Q)$ such that $g_{10}=\mathrm{id},\left.g_{1 t}\right|_{B \times b_{1}(W)}=\mathrm{id}$ for all $t$, $g_{11}\left(K \cap\left(B \times U_{1}\right)\right) \cap\left(L_{1} \cap\left(B \times U_{1}\right)\right)=\varnothing$ and $\left\{g_{1 t}\right\}$ is limited by $\mathcal{U}_{1}$, where $\mathcal{U}_{1}$ is an open cover of $K$ such that $\mathrm{St}^{3}\left(\mathcal{U}_{1}\right)$ refines $\mathcal{U}$. Since $B_{1} \times b_{1}(W)$ is the boundary of $B \times \mathcal{U}_{1}$, we may assume that $\left\{g_{1 t}\right\}$ is an isotopy of $B \times M$ which is the identity outside of $B \times U_{1}$. Applying the same procedure there is an isotopy $\left\{g_{2 t}\right\}$ of $B \times M$ such that $g_{20}=\mathrm{id}, g_{2 t}$ is the identity outside of $B \times U_{2}$ for all $t, g_{21}\left(g_{11}(K) \cap\left(B \times U_{2}\right)\right) \cap\left(L_{1} \cap\left(B \times U_{2}\right)\right)=\varnothing$ and $\left\{g_{2 t}\right\}$ is limited by $\mathcal{U}_{2}$, where $\mathfrak{U}_{2}$ is an open cover of $K$ such that $\mathrm{St}^{3}\left(\mathcal{U}_{2}\right)$ refines $\mathcal{U}_{1}$. Continuing this process we take the isotopy $\left\{g_{1 t}\right\}$ followed by $\left\{g_{2 t}\right\}$, and then followed by $\left\{g_{3 t}\right\}$, etc. We then arrive with a limit isotopy $\left\{g_{t}\right\}$ limited by $\mathcal{U}$ such that $g_{1}(K) \cap L_{1}$ $=\varnothing$. The same idea also enables us to obtain an isotopy for the theorem.

A proof of the general case may be carried out in exactly the same manner as the last part of the proof in [8, Theorem 1$]$.

3. Characterization. Using results of the last section we are able to characterize all fibre $Z$-sets in bundles $(B \times M, p, B)$. A proof may be given using Theorem 2.1 of this paper and Lemma 3.4 of [7] and will be the same as Theorem 1.1 of $[7]$.

Theorem 3.1. Let $E=(B \times M, p, B)$ be a bundle over a polybedron $B$ and let $K \subset E$ be closed. The following are equivalent statements:

(A) $K$ is a fibre Z-set;

(B) $K \cap p^{-1}(b)$ is Q-deficient in eacb $p^{-1}(b)$;

(C) there is an isomorpbism $\phi$ of $B \times M$ such that $\phi(K)$ is an M-projective $Z$-set; and

(D) there is a B-preserving bomeomorphism of $B \times M$ onto $B \times M \times Q$ which carries $K$ into $B \times M \times\{0\}$.

Proof. The same as Theorem 1.1 of [7] (see also the remark above).

\section{Mapping replacement.}

Theorem 4.1. Let $A$ be a locally compact separable metric space and let $X \subset A$ be closed. Suppose $f: A \rightarrow E$ is a proper map into bundle $E=(E, p, B)$ over a polybedron $B$ with fibre $M$ such that $\left.f\right|_{X}: X \rightarrow E$ is a closed imbedding onto a fibre Z-set. Then for any open cover $\mathcal{U}$ of $(A)$ there is a proper imbedding $g: A \rightarrow E$, U-close to $f$, such that $\left.g\right|_{X}=\left.f\right|_{X}, p g(x)=p f(x)$ for all $x$ and $g(A)$ is a fibre Z-set. 
A proof may be given using the following lemma.

Lemma 4.2. Let $X, Y$ be locally compact spaces and let $f: X \rightarrow Y$ be a proper map. Then $f(X)$ is closed. Furthermore, there exists an open cover $\mathcal{U}$ of $Y$ such that if a map $g: X \rightarrow Y$ is U.close to $f, g$ is also proper.

Proof. Elementary.

Proof of Theorem 4.1. In [8, Theorem 4] we prove a similar mapping replacement theorem in the setting of $s$-manifold bundles. The procedure of such a proof, together with Lemma 4.2, may be used here to provide a closed imbedding $g$ for the theorem. In the following we outline the argument.

Let $\left\{V_{i}\right\}$ be a locally finite open cover of $B$ such that $p^{-1}\left(\mathrm{cl}\left(V_{i}\right)\right)$ is trivial for all $i$. Without loss of generality we assume that each $\operatorname{cl}\left(V_{i}\right)$ is itself a polyhedron. Choose a locally finite cover $\left\{A_{k}\right\}$ of $A$ so that each $A_{k}$ is a compact set contained in some member of $\left\{f^{-1} p^{-1}\left(V_{i}\right)\right\}$. Using Lemma 4.2, Theorem 3.1 and the compactness of $A_{1}$ we may prove a similar lemma as Lemma 2 of [8] so that there is a proper map $f_{1}: A \rightarrow E$ satisfying $f_{1}(x)=f(x)$ for $x \in X$, pf $(x)=$ $p f_{1}(x)$ for all $x \in A,\left.f_{1}\right|_{X \cup A_{1}}$ is a closed imbedding whose image is a fibre $Z$-set of $E$ and $f_{1}$ is $\mathcal{U}_{1}$-close to $f$, where $\mathcal{U}_{1}$ is an open cover of $E$ such that $\operatorname{St}^{3}\left(\mathcal{U}_{1}\right)$ refines $U$. By the same manner we may construct proper mappings $f_{2}, f_{3}, \ldots$ of $A$ into $E$ inductively so that, for each $n, f_{n}(x)=f_{n-1}(x)$ for $x \in X \cup A_{1} \cup \cdots \cup$ $A_{n-1},\left.f_{n}\right|_{X \cup A_{1} \cup \ldots \cup A_{n}}$ is a closed imbedding whose image is a fibre $Z$-set of $E$, $p f_{n}=p f$ and $f_{n}$ is ${ }^{\prime} U_{n}^{n}$-close to $f_{n-1}$, where $U_{n}$ is an open cover such that $\mathrm{St}^{3}\left(\mathcal{U}_{n}\right)$ refines $\mathcal{U}_{n-1}$. By virtue of the local finiteness of $\left\{A_{k}\right\}$ and Lemma 4.2, the construction of the mapping $g$ for the theorem clearly follows.

\section{Extending homeomorphisms. Our main result is}

Theorem 5.1. Let $E=(E, p, B)$ be given over a polybedron $B$ with fibre $M$. Suppose $f=\left\{f_{t}\right\}$ is a proper bomotopy of a locally compact separable metric space $A$ into $E$ such that $f_{0}, f_{1}$ are imbeddings of $A$ onto closed fibre Z-sets in $E$ and $p f(\{a\} \times I)=\{$ point $\}$ for all $a \in A$. Then there is an isotopy $\left\{g_{t}\right\}$ on $E$ sucb that $g_{0}=$ id and $g_{1} f_{0}=f_{1}$.

Moreover, if $E$ is trivial, then for any open neighborbood $U$ of $f(A \times I)$ and any open cover $\mathcal{U}$ of $U$ by which $f$ is limited, we may choose $\left\{g_{t}\right\}$ to be a $\left(U, \mathrm{St}^{4}(\mathcal{U})\right)$-isotopy.

Corollary 5.2. Suppose $K_{1}, K_{2}$ are closed fibre Z-sets in $\Delta_{n} \times M$ of bundle $\left(\Delta_{n} \times M, p, \Delta_{n}\right)$ over an $n$-simplex $\Delta_{n}$ and suppose there is a proper bomotopy $f=\left\{f_{t}\right\}$ of $K_{1}$ into $\Delta_{n} \times M$ such that $p f(\{x\} \times I)=\{$ point $\}$ for all $x \in K_{1}, f_{0}=\mathrm{id}$, $f_{t}(x)=x$ for all $t \in I, x \in K_{1} \cap p^{-1}\left(\operatorname{Bd} \Delta_{n}\right)$ and $f_{1}$ is a bomeomorphism of $K_{1}$ 
onto $K_{2}$. Then there is an isotopy $\left\{g_{t}\right\}$ on $B \times M$ sucb that $g_{0}=\mathrm{id}$, $\left.g_{t}\right|_{p-1\left(B d \Delta_{n}\right)}=\mathrm{id}$ for all $t$ and $\left.g_{1}\right|_{K_{1}}=f_{1}$.

To give a proof of the theorem we first establish several special cases designated below as lemmas.

Lemma 5.3. Let $A, B$ be closed $Q$-projective Z-sets of $B \times Q$ and suppose $b: A \rightarrow B$ is a B-preserving homeomorphism. Then $b$ can be extended to $a$ bomeomorphism $H$ of $B \times Q$.

Proof. This follows from the Klee-Anderson techniques while we observe that the requirement for $H$ to be $B$-preserving may be trivially achieved.

Lemma 5.4. Let $A, B$ be metric spaces and let $(B \times Q, p, B)$ be a bundle over $B$. Suppose that (1) $f: A \times I \rightarrow B \times Q$ is a closed imbedding sucb that $p f(\{a\} \times I)=\{$ point $\}$ for all $a \in A$ and $f(A \times I)$ is a Q-projective Z-set; (2) $B_{0}$ is a closed set in $B ;(3) \mathcal{U}$ is a neigbborbood of $f(A \times I)$; and (4) $\phi_{1}, \phi_{2}: B \rightarrow$ $[0,1]$ are maps satisfying $\left.\phi_{1}\right|_{B_{0}}=\left.\phi_{2}\right|_{B_{0}}$. For $i=1$ or 2 , let

$$
C_{i}=\bigcup\left\{f\left(\{a\} \times\left[1 / 2 \phi_{i} p f(a, 0), 1\right]\right): a \in A\right\}
$$

and let $l: C_{1} \rightarrow C_{2}$ be the bomeomorphism such that $f^{-1}$ lf takes each $\{a\} \times$ $\left[1 / 2 \phi_{1} p f(a, 0), 1\right]$, linearly onto $\{a\} \times\left[1 / 2 \phi_{2} p f(a, 0), 1\right]$. Then there is an isotopy $\left\{g_{t}\right\}$ of $B \times Q$ such that $g_{0}=\mathrm{id}, g_{1}$ extends $l$ and for all $t, g_{t}(x)=x$ for any $x \in\left(B_{0} \times Q\right) \cup((B \times Q) \backslash U)$.

Proof. Clearly there is a canonical $B$-preserving homeomorphism $b_{0}$ between $f(A \times I)$ and $f(A \times\{0\}) \times[1 / 3,2 / 3]$ in $(B \times Q) \times[0,1]$ taking each $f(\{a\} \times I\}$ onto $\{f(a, 0)\} \times[1 / 3,2 / 3]$. By Lemma 5.3 we may extend $b_{0}$ to a $B$-preserving homeomorphism $b$ of $B \times Q$ onto $B \times Q \times I$. Now by employing a technique similar to Lemma 4.7 of $[5]$ we can obtain an isotopy $\left\{g_{t}^{\prime}\right\}$ on the bundle $(B \times Q \times I, p, B)$ which uses motions only in the $I$-direction so that the isotopy $\left\{g_{t}=b^{-1} g_{t}^{\prime} b\right\}$ fulfills all the requirements of the lemma.

We now assert a stronger version of Lemma 5.4.

Lemma 5.5. Suppose in Lemma 5.4 we assume that $A$ is separable and locally compact. Then Lemma 5.4 bolds when " $Q$ " is replaced by $Q$-manifold "M".

Let $a \in A$ be any point. Since $p f(\{a\} \times I)=\{$ point $\}, f(\{a\} \times I)$ is contained in the $Q$-manifold $M_{a}=p^{-1}(p f(\{a\} \times I))$. By Lemma 4.1 of [3] there is an imbedding $b_{a}: Q \rightarrow M_{a}$ such that $b_{a}(Q \backslash K)$ is an open set in $M_{a}$ containing $f(\{a\} \times I)$, where $K=\left\{\left(x_{i}\right) \in Q: x_{1}=1\right\}$. 
Since $f: A \times I \rightarrow B \times M$ is an imbedding, using the above remark and the fact $A$ is $\sigma$-compact, it is an elementary exercise to show that there are open covers $\left\{U_{i}\right\}$ of $A$ and $\left\{V_{i} \times b_{i}(Q \backslash K)\right\}$ of $f(A \times I)$, where each $V_{i}$ is open in $B$, such that $f\left(\operatorname{cl}\left(U_{i}\right) \times I\right) \subset V_{i} \times b_{i}(Q \backslash K) \subset U$ for all $i$ and the collection $\left\{V_{i} \times b_{i}(Q \backslash K)\right\}$ is star-finite.

Let $j: V_{i} \rightarrow B$ be the inclusion map. Denote $j \times b_{i}: V_{i} \times Q \rightarrow B \times M$ by $H_{i}$. We may assume the collection $\left\{H_{i}\left(V_{i} \times(Q \backslash K)\right)\right\}$ has been ordered as in Theorem 2 of Anderson-Henderson-West [4]. Choose a closed cover $\left\{A_{i}\right\}$ of $A$ so that each $A_{i} \subset U_{i} \cdot$

Let $\lambda_{1}: A \rightarrow[0,1]$ be a map satisfying $\lambda_{1}(a)=1$ for $a \in A_{1}$ and $\lambda_{1}(a)=0$ for $a \notin U_{1}$. Let

$$
D_{1}=\bigcup\left\{f\left(\{a\} \times\left[1 / 2 \phi_{1} p f(a, 0), 1\right]\right): a \in A\right\}
$$

and

$$
E_{1}=\bigcup\left\{f\left(\{a\} \times\left[1 / 2 b_{1}(a), 1\right]\right): a \in A\right\}
$$

where $b_{1}(a)=\left(1-\lambda_{1}(a)\right) \phi_{1} p f(a, 0)+\lambda_{1}(a) \phi_{2} p f(a, 0)$. Let $l_{1}$ be the natural homeomorphism of $D_{1}$ onto $E_{1}$ so that $f^{-1} l_{1} f$ takes $\{a\} \times\left[1 / 2 \phi_{1} p f(a, 0), 1\right]$ linearly onto $\{a\} \times\left[1 / 2 b_{1}(a), 1\right]$. Note that $D_{1}^{\prime}=H_{1}^{-1}\left(D_{1} \cap H_{1}(B \times Q)\right)$ and $E_{1}^{\prime}=$ $H_{1}^{-1}\left(E_{1} \cap H_{1}(B \times Q)\right)$ are closed $Q$-projective $Z$-sets in $B \times Q . l_{1}$ induces a a homeomorphism of $D_{1}^{\prime}$ onto $E_{1}^{\prime}$. It follows from Lemma 5.4 that there is an isotopy $\left\{g_{1 t}\right\}$ of $B \times M$ so that $g_{10}=\mathrm{id}, g_{11}$ extends $l_{1}$ and $g_{1 t}(a)=a$ for any $a \in\left(B_{0} \times M\right) \cup\left((B \times M) \backslash H_{1}\left(V_{1} \times(Q \backslash K)\right)\right)$.

Let $\lambda_{2}: A \rightarrow[0,1]$ be a map so that $\lambda_{2}(a)=1$ for $a \in A_{2}$ and $\lambda_{2}(a)=0$ for $a \notin U_{2}$. Let

$$
D_{2}=E_{1} \text { and } E_{2}=\bigcup\left\{f\left(\{a\} \times\left[1 / 2 b_{2}(a), 1\right]\right): a \in A\right\}
$$

where $b_{2}(a)=\left(1-\lambda_{2}(a)\right) b_{1}(a)+\lambda_{2}(a) \phi_{2} p f(a, 0)$. Similarly there is an isotopy $\left\{g_{2 t}\right\}$ of $B \times M$ so that $g_{20}=\mathrm{id}, f^{-1} g_{21} f$ takes $\{a\} \times\left[1 / 2 b_{1}(a), 1\right]$ linearly onto $\{a\} \times\left[1 / 2 b_{2}(a), 1\right]$ and $g_{2 t}(a)=a$ for any $a \in\left(B_{0} \times M\right) \cup\left((B \times M) \backslash H_{2}\left(V_{2} \times(Q \backslash K)\right)\right)$. Construct $\left\{g_{3 t}\right\},\left\{g_{4 t}\right\}, \cdots$ analogously. Let $\left\{g_{t}\right\}$ be the limit isotopy obtained by taking $\left\{g_{1 t}\right\}$ followed by $\left\{g_{2 t}\right\}$ and then followed by $\left\{g_{3 t}\right\}$, etc. By the ordering of $\left\{H_{i}\left(V_{i} \times(Q \backslash K)\right)\right\},\left\{g_{t}\right\}$ exists and is an isotopy of $B \times M$ fulfulling all the requirements of the lemma.

Proof of Theorem 5.1. First suppose that $f_{0}(A) \cap f_{1}(A)=\varnothing$. Let us give a proof to the first part of Theorem 5.1. By Theorem 4.1 we may suppose that $f$ is a closed imbedding. Without loss of generality we may also suppose that $B$ is a countable lfsc. Clearly there is a star-finite open cover $\left\{V_{i}\right\}$ and a closed cover $\left\{B_{i}\right\}$ of $B$ such that each pair $\left(V_{i}, B_{i}\right)$ is a simplicial pair $\left(B_{i} \subset V_{i}\right)$ and $p^{-1}\left(V_{i}\right)$ 
is trivial. Let $\phi_{1}: B \rightarrow[0,1]$ be a map such that $\phi_{1}(b)=1$ for $b \in B_{1}$ and $\phi_{1}(b)=0$ for $b \notin V_{1}$. Let

$$
C_{1}=f(A \times I) \text { and } D_{1}=\bigcup\left\{f\left(\{a\} \times\left[1 / 2 \phi_{1} p f(a, 0), 1\right]\right): a \in A\right\} .
$$

Since $f\left(\{a\} \times\left[1 / 2 \phi_{1} p f(a, 0), 1\right]\right)=f(\{a\} \times[0,1])$ for any $a \in A$ such that $p f(a, 0)$ $\notin V_{1}$, and since $p^{-1}\left(V_{1}\right)$ is trivial, by virtue of Theorem 3.1 and Lemma 5.5 we can obtain an isotopy $\left\{g_{1 t}\right\}$ on $E$ such that $g_{10}=$ id and $f^{-1} g_{11} f$ takes each $\{a\} \times I$ linearly onto $\{a\} \times\left[1 / 2 \phi_{1} p f(a, 0), 1\right]$. In particular, $g_{11} f(a, 0)=1 / 2$ whenever $f(a, 0) \in p^{-1}\left(B_{1}\right)$.

Let $\phi_{2}: B \rightarrow[0,1]$ be a map such that $\phi_{2}(b)=1$ for $b \in B_{2}$ and $\phi_{2}(b)=0$ for $b \notin V_{2}$. Let

$$
C_{2}=D_{1} \text { and } D_{2}=\bigcup\{f(\{a\} \times[1 / 2 r(a), 1]): a \in A\}
$$

where $r(a)=\left(1-\phi_{2} p f(a, 0)\right) \phi_{1} p f(a, 0)+\phi_{2} p f(a, 0)$. Similarly there is an isotopy $\left\{g_{2 t}\right\}$ on $E$ such that $g_{20}=$ id and $f^{-1} g_{21} f$ takes $\{a\} \times\left[1 / 2 \phi_{1} p f(a, 0), 1\right]$ linearly onto $\{a\} \times[1 / 2,(a), 1]$. Note that $g_{21} g_{11} f(a, 0)=1 / 2$ whenever $f(a, 0) \epsilon$ $p^{-1}\left(B_{1} \cup B_{2}\right)$.

Continuing this process we take the isotopy $\left\{g_{1 t}\right\}$ followed by $\left\{g_{2 t}\right\}$, and then by $\left\{g_{3, t}\right\}$, etc. We then arrive with a limit isotopy $\left\{g_{t}^{\prime}\right\}$ on $E$ such that $g_{0}^{\prime}=$ id and $g_{1}^{\prime} f_{0}=f_{1 / 2}$. The isotopy $\left\{g_{t}\right\}$ for the theorem clearly follows.

To prove the second part of Theorem 5.1 we may assume, by means of Theorem 4.1, that $f$ is a closed imbedding limited by $\mathrm{St}(\mathcal{U})$. Since $E$ is trivial, we may consider $E$ as $B \times M$. Using the first part of Theorem 5.1 there is a $B$ preserving homeomorphism $b: B \times M \rightarrow B \times M \times[0,1]$ which takes each $f(\{a\} \times l)$ linearly onto $f(a, 0) \times[1 / 3,2 / 3]$. Let $U_{1}=b(U)$ and $\mathcal{U}_{1}$ denote the open cover of $U_{1}$ induced by $\mathcal{U}$ and $b$. Now by employing a technique of Anderson-McCharen we can obtain a $\left(U_{1}, \mathcal{U}_{1}\right)$-isotopy $\left\{g_{t}^{\prime}\right\}$ on the bundle $E^{\prime}=\left(B \times M \times I, p^{\prime}, B\right)$ using motions only in the 1 -direction so that $g_{0}^{\prime}=$ id and $\left(b^{-1} g_{1}^{\prime} b\right) f_{0}=f_{1}$. The isotopy $\left\{g_{t}=b^{-1} g_{t}^{\prime} b\right\}$ is what we wanted.

For the general case suppose that $f_{0}(A) \cap f_{1}(A) \neq \varnothing$. By Theorem 2.1 there is an isotopy $\left\{\lambda_{t}\right\}$ on $E$ (limited by $\mathcal{U}$ when $E$ is trivial) so that $\lambda_{0}=\mathrm{id}$ and $\lambda_{1}\left(f_{0}(A)\right) \cap f_{1}(A)=\varnothing$. Thus we may use the special case above to construct an isotopy $\left\{\mu_{t}\right\}$ on $E$ (limited by $\mathrm{St}^{2}(\mathcal{U})$ when $E$ is trivial) so that $\mu_{0}=$ id and $\mu_{1} \lambda_{1} f_{0}=f_{1} .\left\{\lambda_{t}\right\}$ followed by $\left\{\mu_{t}\right\}$ clearly gives an isotopy for the theorem.

\section{REFERENCES}

1. R. D. Anderson, Topological properties of the Hilbert cube and the infinite product of open intervals, Trans. Amer. Math. Soc. 126 (1967), 200-216. MR 34 \#5045.

2. - On topological infinite deficiency, Michigan Math. J. 14 (1967), 365383. MR $35 \# 4893$. 
3. R. D. Anderson and T. A. Chapman, Extending homeomorphisms to Hilbert cube manifold, Pacific J. Math 38 (1971), 281-293.

4. R. D. Anderson, David W. Henderson and James E. West, Negligible subsets of infinite-dimensional manifolds, Compositio Math. 21 (1969), 143-150. MR 39 \#7630.

5. R. D. Anderson and John D. McCharen, On extending homeomorphisms to Fréchet manifolds, Proc. Amer. Math. Soc. 25 (1970), 283-289. MR 41 \#2711.

6. T. A. Chapman, Dense sigma-compact subsets of infinite-dimensional manifolds, Tran s. Amer. Math. Soc. 154 (1971), 399-426. MR 44 \# 1058.

7. R. Y. T. Wong, On homeomorphisms of in finite-dimensional bundles. I, Trans. Amer. Math. Soc. 191 (1974, 245-259.

8. T. A. Chapman and R. Y. T. Wong, On homeomorphisms of infinite dimensional bundles. II, Trans. Amer. Math. Soc. 191 (1974), 261-268.

SCHOOL OF MATHEMATICS, THE INSTITUTE FOR ADVANCE STUDY, PRINCETON, NET JERSEY 08540

DEPARTMENT OF MATHEMATICS, UNIVERSITY OF CALIFORNIA, SANTA BARBARA, CALIFORNIA 93106 